\title{
¿Valores políticos o desempeño institucional? Un análisis de los determinantes de la confianza en las instituciones políticas en Perú
}

\section{Alexander Benites}

Recibido: 09-09-21

Aprobado: 22-11-21

doi: $10.46476 /$ ra.v2i2.116

\section{Resumen}

Diversos trabajos en la literatura califican la confianza en las instituciones como un elemento crucial para el adecuado funcionamiento del régimen democrático. A pesar de ello, el caso peruano presenta resultados alarmantes en lo que respecta a esta forma de confianza: una abrumadora mayoría de ciudadanos y ciudadanas desconfía de sus instituciones. En ese marco, este trabajo se pregunta, precisamente, por los determinantes de la confianza en las instituciones políticas en el Perú, recurriendo a los valores democráticos y al desempeño percibido sobre el funcionamiento de estos mismos objetos como posibles explicaciones. Los hallazgos de esta investigación muestran que el desempeño institucional juega un rol importante al momento de entender estas variaciones, con énfasis en el sentido de representación como indicador de calidad del proceso político. De forma similar, la idea de un funcionariado público ligado a la corrupción impacta negativamente en la confianza depositada en varias instituciones políticas; mientras que la percepción de un adecuado manejo de la economía nacional eleva los niveles de confianza, siendo estos aún mayores en el caso del presidente de la república. Por el lado de los valores democráticos, los resultados no son concluyentes: si bien el apoyo a la democracia impacta positivamente en la confianza, los indicadores de tolerancia política solo presentan impactos positivos cuando se trata de instituciones relacionadas directamente con el uso de la fuerza.

Palabras clave: confianza en instituciones, Perú, desempeño institucional, valores políticos, cultura política, opinión pública 


\section{Abstract}

Several works in the literature have described political trust as a crucial element for the proper functioning of the democratic regime. In spite of this, the Peruvian case presents alarming results regarding this form of trust: an overwhelming majority of citizens distrust their institutions. In this context, this paper asks precisely for the determinants of trust in political institutions in Peru, resorting to democratic values and the perceived performance of these same objects as possible explanations. The findings of this research show that institutional performance plays an important role in understanding these variations, with emphasis on the sense of representation as an indicator of the quality of the political process. Similarly, the idea of a civil service linked to corruption has a negative impact on the trust placed in various political institutions, while the perception of an adequate management of the national economy raises levels of trust, which are even higher in the case of the president of the republic. On the side of democratic values, the results are not conclusive: although support for democracy has a positive impact on trust, indicators of political tolerance only show positive impacts when it comes to institutions directly related to the use of force.

Keywords: political trust, trust in institutions, Peru, institutional performance, political values, political culture, public opinion

\section{Resumo}

Vários trabalhos na literatura descreveram a confiança nas instituições como um elemento crucial para o bom funcionamento do regime democrático. Apesar disto, o caso peruano apresenta resultados alarmantes em termos desta forma de confiança: uma esmagadora maioria esmagadora de cidadãos desconfia das suas instituições. Neste contexto, este documento pergunta precisamente sobre os determinantes da confiança nas instituições políticas no Peru, recorrendo aos valores democráticos e à percepção do desempenho destes mesmos objectos como possíveis explicações. Os resultados desta investigação mostram que o desempenho institucional desempenha um papel importante na explicação destas variações, com ênfase no sentido da representação como indicador da qualidade do processo político. Do mesmo modo, a ideia de uma função pública ligada à corrupção tem um impacto negativo na confiança depositada em várias instituições políticas, enquanto a percepção de uma gestão adequada da economia nacional aumenta os níveis de confiança, que são ainda mais elevados no caso do presidente da república. Do lado dos valores democráticos, os resultados são inconclusivos: enquanto o apoio à democracia tem um impacto positivo na confiança, os indicadores de tolerância política só mostram impactos positivos quando se trata de instituições directamente relacionadas com o uso da força.

Palavras-chave: confiança nas instituições, Peru, desempenho institucional, valores políticos, cultura política, opinião pública. 


\section{Introducción}

En la actualidad, los bajos niveles de confianza declarados por la ciudadanía en sus instituciones políticas es un fenómeno observado en diferentes partes del mundo (Catterberg y Moreno, 2006; Norris, 2011). A pesar de que el soporte que las y los ciudadanos le otorgan a estos objetos es un elemento central para el adecuado funcionamiento de un régimen democrático (Almond y Verba, 1963; Putnam, 2000; Paramio, 2015), y que muchas de estas instituciones se encuentran en el centro de las democracias liberales (Boidi, 2009), la caída en los niveles de confianza institucional es una problemática a la que se enfrentan tanto sistemas políticos más consolidados (Torcal, 2014) como aquellos con un mayor grado de fragilidad institucional (Carrión, Zárate, Boidi y Zechmeister, 2020).

Insertado en esta tendencia, sin embargo, el caso de Perú es particularmente alarmante. Este último, según lo reportado por diferentes sondeos de opinión, se encuentra en los peldaños más bajos de la región en lo que respecta a confianza en las instituciones, y presenta resultados igual de preocupantes en otros indicadores que evalúan la relación entre el ciudadano y el sistema político. Así, por ejemplo, no solo el grado de confianza en los partidos políticos o el Congreso de la República muestran los niveles más bajos de toda América Latina, sino que el país reporta, a su vez, altos niveles de tolerancia frente a golpes militares, despreocupación por el equilibrio de poderes y alta insatisfacción con el funcionamiento de la democracia (Carrión, Zárate, Boidi y Zechmeister, 2020).

Es en ese marco que surgen algunas interrogantes: ¿qué se encuentra detrás de la ausencia de confianza en las instituciones en el país? O, de forma similar, ¿cuáles son los factores que podrían dar luces sobre cómo mejorar tan bajos niveles de confianza? Esta investigación se pregunta, precisamente, por los determinantes de las variaciones individuales en los niveles de confianza en las instituciones políticas en Perú. Y, para responder a dicha interrogante, recurre a dos vertientes explicativas, identificadas en la literatura como fuentes originarias de la confianza institucional: el desempeño de estos mismos objetos políticos y los valores democráticos.

Explorar los determinantes de esta forma de confianza es de suma relevancia si se toma en cuenta el consenso que hoy existe sobre la importancia del indicador para el adecuado funcionamiento del régimen político. En esa línea, una variedad de trabajos ha mostrado cómo aquellos ciudadanos y ciudadanas que poseen mayores niveles de confianza institucional también presentan una mayor propensión a cumplir la ley (Marien y Hooghe, 2011), mayores niveles de participación electoral (Hooghe, Marien y Pauwels, 2011) y una menor tendencia a apoyar propuestas políticas extremistas (Hooghe y Marien, 2013; van Stekelenburg y Klandersman, 2018). La propuesta teórica detrás de estos hallazgos es que la confianza en las instituciones tiene impactos tangibles en el funcionamiento de la democracia en tanto refleja, en algún punto, el grado de legitimidad que goza el proceso político (Almond y Verba, 1963, p. 230) y el apoyo al ordenamiento democrático en un sentido más general (Easton, 1975). 
La confianza institucional, además, juega un rol igual de importante - aunque muchas veces pase desapercibido - en la puesta en marcha de reformas a diferente nivel. Si las instituciones de gobierno carecen de legitimidad y soporte social desde la ciudadanía, el apoyo necesario para implementar reformas sustantivas es difícil de movilizar, más aún cuando ello involucra sacrificios de corto plazo y los resultados son poco tangibles en el largo plazo (OECD, 2013). Esto es central en el caso peruano, uno que requiere reformas urgentes, pero posee importantes limitaciones para llevarlas a cabo: élites económicas y políticas desinteresadas y un sistema que se defiende de los esfuerzos al cambio (Dargent, 2021). Es en dicho escenario que preguntarse por sus determinantes se vuelve fundamental.

En lo que respecta a la primera parte del argumento, se evalúa la percepción del manejo de la economía nacional, el grado de receptividad de las autoridades políticas y el grado de corrupción percibida como indicadores que miden el desempeño institucional a nivel de resultados y de proceso político. Esta forma de explicar la confianza ha sido catalogada como «endógena», en tanto es producida por y al interior del sistema político. Así, la confianza en las instituciones se configura como el cálculo racional que los individuos realizan sobre la manera en la cual estos objetos políticos se encuentran operando (Mishler y Rose, 2001).

En un segundo plano, se plantea que los valores políticos asociados a la democracia tienen impactos positivos en los niveles de confianza institucional. Desde la literatura, se ha identificado a esta forma de confianza como una expresión de la preferencia por el ordenamiento político democrático (Easton, 1975), y ha sido posicionada como un indicador intermedio entre el cálculo racional sobre lo que el régimen es capaz de ofrecer y la preferencia por la democracia como forma de gobierno (Rohrschneider y Schmitt-Beck, 2002). Se espera, así, que aquellas personas con valores asociados a la democracia también muestren mayores niveles de confianza institucional. Para ello, se toma el apoyo a la democracia y el apoyo a la participación política de personas con opiniones contrarias a las del gobierno como elementos representativos de los valores democráticos.

Este trabajo se inserta dentro del enfoque de análisis micro-fundacional de las teorías sobre confianza institucional, planteando que esta última varía entre los individuos a partir de las diferentes evaluaciones y experiencias que desarrollan en la interacción con sus sistemas políticos respectivos (Levi y Stroker, 2000; Mishler y Rose, 2001). Como insumos para validar las hipótesis propuestas, y utilizando una metodología de corte cuantitativo, se recurre a los datos de la encuesta del Barómetro de las Américas, recopilados por el Latin American Public Opinión Project (LAPOP), entre los años 2010 y 2019.

Más allá de los resultados específicos, esta investigación busca brindar dos contribuciones principales. Por un lado, utilizar los marcos teóricos existentes para 
dar cuenta de los elementos que podrían ayudar a comprender la variación en los niveles de confianza institucional para el caso peruano. En ese proceso, además, analizar si es que los valores y las percepciones individuales se complementan en los eventuales impactos que tendrían sobre esta forma de confianza, o si se comportan de manera independiente, una temática de interés desde los estudios sobre cultura política. Y, por último, aproximarse a la forma en que los determinantes de estos niveles de confianza se vuelven heterogéneos (o no) al estudiar dichas asociaciones para cada institución en específico, dando, así, mayores luces sobre las dinámicas de interacción que existen entre la ciudadanía y el sistema político en el caso de Perú.

\section{El caso peruano}

Perú es un caso sumamente preocupante de confianza en las instituciones. Por mencionar algunas cifras, según los datos recopilados por el Barómetro de las Américas, en el año 2019, el país presenta los niveles más bajos de confianza en instituciones como los partidos políticos (7\%) o la legislatura nacional (9\%) de toda la región latinoamericana. Con algunos matices, la legitimidad que gozan varios objetos políticos cruciales para el adecuado funcionamiento de un régimen democrático se mantiene siempre por debajo del promedio regional, y en algunos casos muestra una tendencia a la caída. Los niveles de confianza en las instituciones a nivel histórico se pueden observar en el Gráfico $\mathrm{N}^{\circ} 1$.

Gráfico No1: Confianza en las instituciones en Perú y América Latina
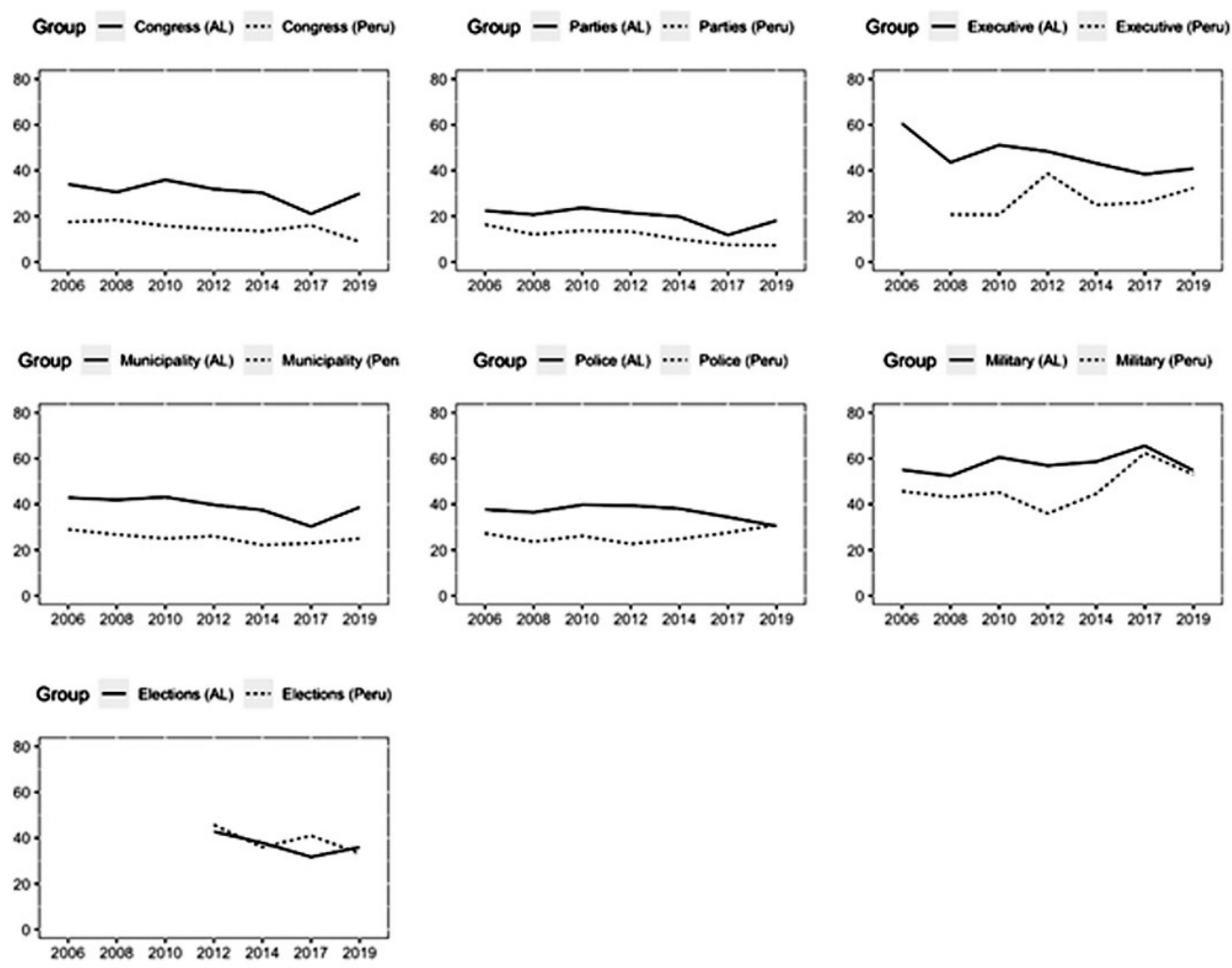

Elaboración propia. Fuente: Barómetro de las Américas (2006-2019) 
Estos resultados son una parcela del grado de desafección con la política que hoy existe en Perú. Cifras adicionales del Barómetro de las Américas también muestran que el caso se encuentra en su más bajo nivel de apoyo a la democracia a nivel histórico: menos de la mitad de las y los ciudadanos peruanos (48\%) declara preferir a la democracia como régimen político a pesar de sus problemas. De manera similar, el 66\% de las y los peruanos consideran que las autoridades políticas no se encuentran interesadas en sus necesidades y demandas, más de la mitad cree justificado que los militares tomen el poder cuando hay mucha delincuencia o mucha corrupción, y seis de cada diez peruanos/as encuentran adecuado que el presidente cierre el Congreso y gobierne sin este poder del Estado en momentos difíciles (Carrión, Zárate, Boidi y Zechmeister, 2020). Todos ellos, indicadores asociados directamente a la confianza en las instituciones, según lo discutido por varios trabajos en la literatura (Hooghe, Marien y Pauwels, 2011; Hooghe y Marien, 2013; van Stekelenburg y Klandersman, 2018; Mauk, 2020).

A pesar de los esfuerzos, las explicaciones a este fenómeno en Perú siguen siendo un tema poco explorado por la disciplina. Concretamente, en el caso de la confianza institucional, algunas investigaciones han buscado dar respuesta a los determinantes de esta forma de confianza incluyendo a Perú como parte de un conjunto más grande de países en los cuales se testean ciertas hipótesis (Catterberg y Moreno, 2006; Freitag y Bühlman, 2009; Mauk, 2020). Sin embargo, los esfuerzos por tratar de explicar los bajos niveles de confianza institucional que presenta el caso peruano, de manera particular, son mucho menores.

Sobre lo último, Levitt (2011) y Cruz y Guibert (2013) exploran los determinantes de la confianza en el Congreso de la República. En el caso del primero, se pone a prueba la manera en que la sintonía entre la autonomía esperada y la observada en la legislatura nacional eleva los niveles de confianza en dicha institución. De manera complementaria, Levitt identifica impactos poco significativos de variables sociodemográficas y al desempeño económico como uno de los predictores más altos de la confianza en el Congreso de la República (Levitt, 2011). En el segundo caso, Cruz y Guibert buscan explicar la confianza en el Congreso a partir de variables sociodemográficas, actitudinales y de desempeño institucional. Los hallazgos muestran que las personas que viven en la capital presentan menores niveles de confianza, mientras que la identificación partidaria los eleva. Y, por último, que la percepción de un buen desempeño del presidente de la república y un buen desempeño percibido sobre el accionar de los congresistas tienen impactos positivos en la confianza en el Congreso (Cruz y Guibert, 2013).

Finalmente, el trabajo de Arcaya (2016) explora las variables que tienen una mayor influencia a la hora de explicar los niveles de confianza en cuatro instituciones: el Congreso de la República, el gobierno regional, la policía y el Poder Judicial. Aunque su metodología es innovadora, la principal conclusión es que mayores 
niveles educativos elevarán los niveles de confianza en las instituciones políticas (Arcaya, 2016). Dicha relación ha sido bastante contestada desde otros trabajos (Norris, 2011), mostrando que la interacción entre ambas variables es mucho más compleja y depende de otros elementos, tales como el grado de corrupción (objetivo o percibido) del sistema político y los objetos que lo componen (Hakhverdian y Mayne, 2012).

Como se observa, a pesar de los alarmantes niveles de confianza institucional que presenta el caso peruano, y la importancia que diferentes estudios le otorgan al indicador tanto a nivel teórico como empírico, persiste la ausencia de investigaciones que den cuenta de los determinantes de la confianza institucional en Perú. Peor aún, los trabajos ya desarrollados no parten de lentes teóricos ya esbozados por la disciplina, y tampoco son capaces de identificar las variaciones (o su ausencia) en el argumento para diferentes tipos de instituciones, ya que generalmente se centran específicamente en una sola. En dicho marco, esta investigación buscar aproximarse a algunas de las interrogantes planteadas.

\section{La confianza en las instituciones y sus determinantes}

La confianza es, fundamentalmente, una evaluación. Como concepto, hace referencia a la expectativa de un actor A sobre la realización de una acción o conjunto de acciones por parte de un segundo actor B. En ese sentido, es relacional y da cuenta de la expectativa de A sobre el accionar de B, expresando un juicio valorativo sobre el grado de fiabilidad del segundo actor en función al primero (Hardin, 1996; Levi, 1996; Levi y Stroker, 2000). Cuando se hace referencia a la confianza en las instituciones de manera particular, esta expresa el juicio valorativo que las y los ciudadanos realizan sobre el grado de fiabilidad de los objetos que componen sus sistemas políticos: las instituciones, agencias del Estado y los actores que las conforman (van der Meer, 2017; van der Meer y Hakhverdian, 2017).

Desde la literatura, se han planteado, al menos, tres formas de explicar la confianza en las instituciones (van der Meer, 2017; van der Meer y Hakhverdian, 2017). Por un lado, se encuentran las explicaciones centradas en las características de los objetos políticos portadores de la confianza, haciendo referencia a las propiedades de los actores e instituciones que componen al sistema, al igual que sus niveles de desempeño en diferentes esferas. Este tipo de explicaciones han sido también denominadas «endógenas», en tanto se entiende a la confianza en las instituciones como un resultado producido por y al interior del sistema político. Por el otro lado, se encuentran las explicaciones enfocadas en las características del sujeto que emite este juicio valorativo, prestando atención a sus procesos de socialización, predisposiciones genéticas, actitudes y valores. Este tipo de explicaciones se han denominado «exógenas», en tanto los componentes se consideran externos al sistema político (Mishler y Rose, 2001). 


\section{Explicaciones endógenas: cualidades de los objetos políticos y el desempeño institucional}

Al hablar delas explicaciones endógenas y relacionadas a las cualidades delos objetos políticos portadores de confianza, estas recurren a las características del proceso político (input institutions) y al desempeño institucional (institutional output). Sobre lo primero, las características se refieren comúnmente a elementos como elecciones transparentes, representación política y rendición de cuentas. Sobre lo segundo, el indicador más utilizado de desempeño es el manejo macroeconómico de las instituciones (van der Meer, 2017; van der Meer y Hakhverdian, 2017). Sin embargo, sea el proceso político o el desempeño, surgen las interrogantes sobre si estos hacen referencia a las características y el desempeño objetivo de las instituciones, o al percibido (y subjetivo) desde la ciudadanía y cómo ambos impactan en la confianza institucional.

Primero, al analizar el impacto del desempeño y las características objetivas de las instituciones en la confianza, los resultados no son concluyentes. A excepción del impacto negativo que muestran sistemas políticos e instituciones con altos niveles de corrupción en esta forma de confianza (Hakhverdian y Mayne, 2012; van der Meer y Hakhverdian, 2016), otras áreas del desempeño institucional muestran resultados menos claros. En lo relacionado al desempeño económico objetivo de las instituciones, por ejemplo, algunos trabajos encuentran una relación positiva entre este y la confianza en las instituciones (Miller y Listhaug, 1999; Taylor, 2000), al igual que efectos negativos causados por periodos de recesión (Stevenson y Wolfers, 2011) y niveles elevados de desigualdad económica (Medve-Bálint y Boda, 2014). No obstante, otras investigaciones no encuentran efectos significativos de buenos indicadores económicos en la confianza institucional (Rahn y Rudolph, 2005; Hakhverdian y Mayne, 2012; van der Meer y Hakhverdian, 2016). En consecuencia, hasta qué punto el desempeño y las características objetivas de las instituciones tienen impactos en la confianza institucional, es todavía un elemento de disputa (van deer Meer y Dekker, 2011; van deer Meer y Hakhverdian, 2016).

Pero, contrario a lo que sucede con el desempeño objetivo, el desempeño percibido de las instituciones muestra impactos mucho más claros y consistentes en la confianza en las instituciones. Diversos trabajos en la materia han mostrado de forma reiterada los impactos positivos que presentan la percepción de un adecuado manejo de la economía nacional (Citrin y Green, 1986; Hetherington y Rudolph, 2008; Mishler y Rose, 2001; van deer Meer y Dekker, 2011; Rohrschneider y Schmitt-Beck, 2002; Levitt, 2011); la capacidad del gobierno para proveer a sus ciudadanos de derechos, libertades y procedimientos justos (Newton y Norris, 2018); la receptividad de las autoridades políticas (Torcal, 2014); y las evaluaciones positivas sobre su funcionamiento en un plano más general (Rohrschneider y Schmitt-Beck, 2002; Catterberg y Moreno, 2006). De forma similar, son bien 
conocidos los efectos negativos que tienen altos niveles de corrupción percibida en las instituciones (Mishler y Rose, 2001; Catterberg y Moreno, 2006; Chang y Chu, 2006; Morris y Klesner, 2010) y la sensación de un mal manejo de la seguridad ciudadana (Blanco Ruiz, 2014).

De la mano con estos hallazgos, diferentes trabajos enfocados en los países latinoamericanos han llegado a conclusiones muy similares. Por ejemplo, Güemes (2015) y Guëmes y Brugué (2016), explorando los determinantes de la confianza institucional para los países de la región, encuentran que esta forma de confianza no depende de indicadores agregados u objetivos de desempeño institucional, sean la independencia y calidad de las burocracias estatales, o el desempeño del sector público en términos de cobertura, acceso a servicios sociales o niveles de alfabetización.

Por el contrario, son las percepciones a nivel individual las que demuestran tener impactos significativos en esta forma de confianza. Así, se identifican impactos positivos de altos niveles de satisfacción con el funcionamiento de la democracia y la percepción de eficiencia del funcionariado público y del accionar de algunas instituciones en específico (Güemes, 2015; Guëmes y Brugué (2016). En esa línea, algunos estudios muestran los impactos negativos que tiene en la confianza institucional percepciones de un alto nivel de desigualdad en la riqueza (Scartascini y Luna, 2020); así como el impacto positivo que existe cuando la ciudadanía considera que las instituciones y autoridades cumplen con sus promesas de gobierno (Alessandro, Lagomarsino, Scartascini, Streb y Torrealday, 2021).

Como resultado, una primera conclusión es que la performance institucional determina en buena parte la confianza que la ciudadanía le otorga a sus instituciones de gobierno (Newton y Norris, 2018, p.12). Este hecho hace sentido si se toma en cuenta que la confianza nunca es una relación incondicional, sino que requiere que aquello sobre lo cual se deposita la confianza demuestre que es un actor u objeto de fiar (Levi y Stroker, 2000). Desde esta perspectiva, la confianza en las instituciones se configura con el cálculo racional que los individuos realizan en función a qué tan bien, dichos objetos políticos se encuentran operando (Hetherington 1998; Mishler y Rose, 2001). Sin embargo, la relación confianza-desempeño es sólida siempre y cuando se refiera al ámbito de las percepciones, ya que los impactos del desempeño y las características objetivas de las instituciones son aún temas de agenda pendiente.

\section{Explicaciones exógenas: las características del potential truster}

Las explicaciones exógenas a la confianza en las instituciones tienen que ver con un conjunto de elementos que se encuentran por fuera del sistema político, y son traídos al mismo por el potential truster. Estos se relacionan a características de la personalidad, los niveles de participación en redes comunitarias y los valores 
políticos. La idea detrás de estas explicaciones es que pueden ser rastreadas a partir de las diferentes interacciones en las que los individuos se encuentran insertados, desde los diferentes círculos sociales en los que se desarrollan (Mishler y Rose, 2001; Newton y Norris, 2018).

En primer lugar, desde el enfoque psicológico-social, se plantea que la confianza es un elemento constitutivo de la personalidad del individuo. Así, algunas personas presentan una mayor predisposición a confiar, cooperar y ayudar a los demás, teniendo mayor confianza en los asuntos públicos, la política y sus instituciones (Newton y Norris, 2018). En consecuencia, existen individuos más optimistas y que tienden a confiar - trusting people-, en comparación con otros más cautos y desconfiados - cynics- (Gabriel, 1995).

En segundo lugar, y de la mano con el enfoque previo, se encuentran las teorías culturales. Desde esta vertiente, se defiende que la habilidad de confiar en otros y cooperar es el resultado, más bien, de procesos de socialización expresados en las diferentes asociaciones voluntarias de individuos (Putnam, 1995; Newton y Norris, 2018). En ese sentido, la confianza entre pares permite la construcción de redes interconectadas, que luego son proyectadas hacia las instituciones (Almond y Verba, 1963). La confianza permite que las instituciones funcionen porque «derrama» estas redes entre las asociaciones fomentando la cooperación, y luego las lleva «hacia arriba» construyendo redes nacionales de instituciones necesarias para la existencia de un gobierno representativo (Putnam, 1995). La institucionalización de interacciones basadas en la confianza genera procesos de dependencia, en los cuales los mecanismos de socialización transmiten esta predisposición - positiva o negativa - hacia las instituciones del sistema político de una generación a otra (Putnam, 2000; Mishler y Rose, 2001).

Ambos argumentos han sido puestos a prueba, al evaluar los impactos que tiene en la confianza institucional el grado de confianza interpersonal de las y los ciudadanos como indicador de esta predisposición. Se esperaba que, a medida que los niveles de confianza en los pares y miembros de la comunidad sea mayor, los niveles de confianza en las instituciones también lo serían. A pesar de ello, los resultados de este indicador son poco concluyentes. Algunos trabajos muestran que la confianza interpersonal y los procesos de socialización no manifiestan, prácticamente, ningún efecto en la confianza institucional (Mishler y Rose, 2001); o que, si existe alguna relación estadística, la asociación es bastante débil y poco consistente (Newton y Norris, 2018).

En un tercer nivel, se encuentran los impactos de los valores democráticos como explicación alternativa a la confianza institucional. Esta vertiente parte de los aportes teóricos de Easton (1965; 1975), en lo relacionado a la distinción entre el apoyo específico y el apoyo difuso al sistema político. Según el autor, la primera forma de apoyo es aquella que la ciudadanía extiende específicamente a las autoridades de turno; tiende a ser más fluctuante y está fuertemente ligada a la evaluación que los 
individuos realizan sobre el desempeño de las mismas. Por el contrario, el apoyo difuso es más estable y difícil de modificar. Este último se extiende no solo hacia las autoridades, sino también hacia las agencias e instituciones políticas, hasta llegar al régimen y la comunidad política en su conjunto. De esa manera, hace referencia a la preferencia que las y los ciudadanos expresan por los elementos subyacentes al ordenamiento político en un sentido más general (Easton, 1965; 1975).

Esta preferencia por el ordenamiento político (democrático), siguiendo a Easton, se expresa en los niveles de legitimidad y confianza que la ciudadanía deposita en aquellos objetos que componen el sistema político, lo que lleva a pensar inevitablemente en las instituciones del mismo (Easton, 1975, p. 447). Si bien el autor es cauto al explicar la dificultad de medir empíricamente el concepto, la literatura ha posicionado a la confianza en las instituciones como un punto intermedio entre el apoyo específico a las autoridades y sus respuestas racionales al desempeño, por un lado, y el apoyo hacia la manera en la cual se ejerce el poder y el ordenamiento político en un sentido más general, por el otro (Norris, 2011; Rohrschneider y Schmitt-Beck, 2002). En suma, se espera que aquellos individuos con una preferencia por el régimen democrático, expresado ello en valores asociados a la democracia, muestren niveles más elevados de confianza institucional.

Uno de los hallazgos más recientes sobre la relación entre los valores democráticos y la confianza institucional, sin embargo, es que depende de manera importante sobre la forma en la cual el sistema democrático se encuentra operando. Desde los trabajos sobre cultura política, se plantea que los valores que poseen las y los ciudadanos sirven como puntos de referencia de lo que estos esperan de su sistema político (Easton, 1965; Fuchs, 2009; Lipset, 1959). Los valores democráticos, por consecuencia, conducirían a mayores niveles de confianza si es que los sistemas políticos alcanzan dichas expectativas. Así, se espera que, en contextos democráticos, los valores asociados a la democracia tengan efectos positivos en la confianza institucional, y el efecto sea inverso en entornos autoritarios (Breustedt y Stark 2015; Catterberg y Moreno, 2006; Singh 2018).

Trabajos recientes han puesto a prueba esta hipótesis, observando que el efecto de los valores democráticos en la confianza institucional, efectivamente, es positivo en contextos democráticos y negativos en contextos autoritarios (Kołczyńska, 2020). En ese mismo sentido, Mauk (2020) identifica que, más que el contexto objetivo, el efecto de los valores democráticos en esta forma de confianza se encuentra mediado por la percepción individual sobre la calidad del régimen político. Esto último se alinea con una de las conclusiones a la que han llegado los trabajos sobre confianza institucional: que su explicación pasa fuertemente por las percepciones individuales, mientras que los impactos de las características objetivas del sistema muestran todavía resultados poco claros (van deer Meer y Dekker, 2011; van deer Meer y Hakhverdian, 2016).

En suma, una conclusión sobre los trabajos que exploran los determinantes de la confianza institucional es que esta es un reflejo de las experiencias que los 
individuos desarrollan en sus contextos políticos, y que la heterogeneidad que existe en los niveles de confianza en las instituciones, en una comunidad política es una muestra de dicha variedad de percepciones (Levi y Stroker, 2000, p. 481). Más aún, en la medida que explicar el fenómeno parte, fundamentalmente, de la experiencia que la ciudadanía desarrolla en la interacción con estos sistemas, ambas formas de explicar la confianza institucional (endógenas y exógenas) no solo no son opuestas, sino que pueden llegar a ser complementarias: ciertas predisposiciones iniciales (que vienen por fuera del sistema) pueden ser constantemente reforzadas o modificadas en función a las evaluaciones repetitivas que se realizan sobre el comportamiento de las instituciones (Mishler y Rose, 2001, p. 38). En consecuencia, ambas deben ser tomadas en cuenta al momento de explorar los determinantes de la confianza en las instituciones.

\section{El argumento}

Identificar los elementos que explican las variaciones en los niveles de confianza institucional pasa, a partir de lo discutido en el apartado anterior, por recurrir a los dos grandes grupos de explicaciones al fenómeno de forma complementaria: las características y cualidades del potential truster, y sus percepciones sobre el desempeño institucional y la calidad del proceso político. En tanto tal, se proponen dos hipótesis:

Hipótesis 1: La percepción de un adecuado desempeño institucional y calidad del proceso político tienen impactos positivos en los niveles de confianza institucional.

En aquellos casos donde se percibe que hay un adecuado manejo de la economía (institutional output), las autoridades son receptivas de las necesidades del ciudadano y se percibe a funcionarios y funcionarias públicas como actores ajenos a casos de corrupción (input institutions). De esta forma, habrá impactos positivos en la confianza en las instituciones.

Hipótesis 2: La presencia de valores democráticos tendrá un impacto positivo en los niveles de confianza institucional.

El apoyo explícito a la democracia a pesar de sus problemas y la presencia de valores asociados, expresado ello en el apoyo al voto y a la manifestación pacífica de personas con opiniones críticas a las del gobierno - ambos indicadores de tolerancia política一, tendrán un impacto positivo en los niveles de confianza institucional. Todo ello en la medida que la confianza en las instituciones, además de una evaluación racional sobre el desempeño de estas, expresa una preferencia por el ordenamiento político democrático (Easton, 1975; Rohrschneider y Schmitt-Beck, 2002).

No obstante, y como es puesto a prueba por Mauk (2020), la asociación entre el apoyo a la democracia y la confianza institucional depende de qué tan bien se considera que el régimen político se encuentra operando. Por ello, en aquellos 
casos donde la percepción es que el régimen político se encuentra operando de forma adecuada, el apoyo a la democracia tendrá efectos positivos en la confianza institucional. Por el contrario, cuando la percepción sea que la democracia se encuentra operando de manera negativa, el apoyo a la democracia tendrá impactos negativos en esta forma de confianza, ya que los valores políticos no encuentran un correlato en la realidad.

\section{Datos y metodología}

Para dar cuenta de los determinantes de la confianza institucional en el Perú, se utilizan los datos recopilados por las encuestas del Barómetro de las Américas, realizadas por el Latin American Public Opinión Project (LAPOP), entre los años 2010 y 2019. Se tiene, así, un marco muestral de 10,168 personas. ${ }^{1}$ Los datos han sido recogidos utilizando diseños de muestreo probabilístico a nivel nacional de todos los adultos en edad de votar, tomando en cuenta estratificación y agrupamiento para su elaboración.

Las hipótesis de esta investigación se ponen a prueba al realizar un total de diez modelos de regresión lineal de mínimos cuadrados (OLS) con efectos de diseño incorporados, divididos en dos grupos. En el caso del primer grupo, se incluyen, primero, las variables independientes; y posteriormente, se agregan las variables de control y los efectos de interacción. En los tres modelos del primer grupo, la variable dependiente es un índice aditivo que representa la sumatoria de siete instituciones del sistema político: el Congreso de la República, los partidos políticos, la figura del presidente de la república, los municipios locales, las fuerzas armadas, la policía y las instituciones electorales. ${ }^{2}$ Todas las variables seleccionadas son de naturaleza ordinal, con valores que oscilan entre 1 y 7 , donde el primero representa un valor muy bajo de confianza y el último un valor muy alto de confianza en dicha institución.

A nivel de predictores, se tiene la variable Desempeño económico, que evalúa la percepción del ciudadano/a sobre qué tan bien considera que las instituciones vienen manejando la economía nacional con respecto al año previo (institutional output). Este es un indicador clásico para evaluar la percepción del desempeño

1. Todos los años de la encuesta han sido unificados en una sola base de datos. En esa línea, se asume que no existe dependencia temporal entre los datos, y que los individuos son capaces de diferenciar el comportamiento institucional del presente con respecto al del pasado. Sin embargo, esta es una limitación metodológica importante, en la medida que se puede plantear que la confianza en las instituciones actual de un individuo $(t)$ es el resultado de la suma ponderada de sus experiencias políticas pasadas (t-1) (Fiorina, 1978). Así, los modelos no son capaces de controlar eventuales dependencias temporales entre las variables.

2. Antes de proceder con el agregado de variables, se utilizaron técnicas de reducción de dimensiones para validar dicha sumatoria de forma estadística. El alpha de Cronbach presentó un valor de .82, lo que otorga solidez a la elaboración del índice aditivo. Este indicador tiene un valor máximo de $43 \mathrm{y}$ uno mínimo de 1. 
institucional en la literatura (van der Meer y Hakhverdian, 2017). Adicional a ello, se encuentran las variables Receptividad política y Corrupción, ambos indicadores relacionados a las características del proceso político (input institutions). En el caso de la primera, evalúa qué tanto la persona encuestada considera que las autoridades políticas se encuentran interesadas en sus demandas y necesidades. En el caso de la segunda, se evalúa qué tanto se considera que las y los funcionarios públicos se encuentran involucrados en casos de corrupción.

En el caso de los valores democráticos, se tienen tres indicadores. Por un lado, el apoyo explícito a la democracia como forma de gobierno preferible a pesar de sus problemas (Democracia). Esta es una variable ordinal del 1 al 7, en la que el primer valor expresa un muy bajo nivel de apoyo, y el último un nivel muy alto de apoyo. De manera complementaria, se incluyen dos indicadores de tolerancia política: qué tanto considera la persona encuestada que las personas con opiniones críticas a las del gobierno tengan derecho a votar (Voto críticos/as) y tengan derecho a manifestarse pacíficamente (Manifestaciones críticos/as). Ambas son variables ordinales con 10 niveles, que van desde un muy bajo hasta un muy alto nivel de apoyo.

Finalmente, sobre los valores democráticos, se pone a prueba la propuesta de Mauk (2020), quien plantea que la asociación positiva entre el apoyo a la democracia y la confianza en las instituciones depende de qué tan bien (la persona considera) el régimen se encuentra operando. Por ello, en un tercer nivel de análisis, se incluyen efectos de interacción en los modelos de regresión, con el objetivo de poner a prueba esta hipótesis, incluyendo el indicador Satisfacción con la democracia, variable ordinal de cuatro niveles recodificados, donde 1 indica un muy bajo nivel de satisfacción y 4 un muy alto nivel de satisfacción con el régimen político.

Las variables de control incluidas en los modelos de regresión tienen que ver, centralmente, con el nivel de confianza que la persona encuestada tiene en sus pares (Confianza interpersonal), argumento ampliamente discutido por la literatura (Putnam, 2001; Mishler y Rose, 2001). Además, se incluyen como controles la posición en la que la o el ciudadano se ubica en el eje izquierda-derecha del espectro político, el último nivel de estudios alcanzado, la edad, el género y si la persona encuestada reside en una zona rural o en una zona urbana.

En el segundo grupo de modelos de regresión, los predictores son los mismos, pero las variables dependientes son las instituciones por separado, con el objetivo de evaluar cómo varía (o no) el argumento según el tipo de institución. Debido a la dificultad para interpretar los efectos principales (main effects) de las variables independientes sobre las dependientes cuando hay efectos de interacción en los modelos de regresión, el indicador Satisfacción con la democracia ha sido incluido como una variable de control en este grupo. Sin embargo, los modelos con efectos de interacción se encuentran en los anexos del documento. Por último, y para una mayor facilidad en la comparación entre predictores, debido a que las variables independientes tienen diferentes unidades de medición, los coeficientes han sido estandarizados, evaluando los cambios en desviaciones estándar sobre la variable dependiente. 
Una salvedad metodológica antes de iniciar con la discusión de resultados. Es importante reconocer y mencionar los problemas y limitaciones con las que debe lidiar este trabajo. En primer lugar, se debe tomar en cuenta la posibilidad de causalidad inversa en la relación entre, por ejemplo, confianza y desempeño institucional: podría llegar a darse el caso de que la confianza en las instituciones sea la que determine la existencia de una adecuada percepción sobre el desempeño de estos mismos objetos. De forma similar, otra limitación se encuentra relacionada a la posibilidad de omisión de variables en los modelos propuestos. Al ser la confianza un resultado de las evaluaciones y experiencias que los individuos desarrollan en sus respectivos contextos políticos (Levi y Stroker, 2000), la cantidad de elementos que podrían influenciar la relación causal es amplia, siendo perfectamente posible omitir alguno de ellos.

Mitigar ambos puntos, sin embargo, conllevaría la construcción de modelos poco parsimoniosos, con una cantidad sustancialmente mayor de variables que podría dificultar la interpretación de resultados. De manera similar, lidiar con la posibilidad de causalidad inversa requeriría incorporar data panel en el análisis o un diseño metodológico experimental, dos caminos poco factibles. Por ello, el trabajo descansa en el constructo teórico desarrollado por la literatura al momento de plantear la relación causal entre variables, al igual que la selección de predictores y variables de control. Aun así, las interpretaciones enarboladas a partir de los resultados deben ser leídas con cautela, tomando en cuenta sus limitaciones y deficiencias metodológicas.

\section{Resultados}

\section{Explicando la confianza en las instituciones en Perú}

En esta sección se explora los determinantes de la confianza en las instituciones para el caso peruano. Empezando por los impactos del desempeño institucional, los resultados presentados en la Tabla $\mathrm{N}^{\circ} 1$ muestran que la percepción de un adecuado desempeño de las instituciones en el manejo de la economía nacional (Desempeño económico) tiene impactos positivos y estadísticamente significativos en el nivel de confianza institucional. Estos impactos son consistentes a lo largo de los tres modelos. Y, en comparación con todos los demás predictores, es una de las variables con mayor efecto sobre el nivel de confianza en las instituciones.

Como lo muestra el Modelo 2, el aumento de un punto en desviaciones estándar de la variable independiente genera el aumento de .118 desviaciones estándar sobre la variable dependiente manteniendo todos los demás regresores constantes. Así, el impacto del desempeño percibido de las instituciones en términos de manejo económico sobre la confianza en estos objetos políticos, para el caso peruano, se encuentra alineado con los hallazgos de buena parte de la literatura sobre la materia (Citrin y Green, 1986; Hetherington y Rudolph, 2008; Mishler y Rose, 2001; van deer Meer y Dekker, 2011; van deer Meer, 2017; Rohrschneider y Schmitt-Beck, 2002; Levitt, 2011). 
Tabla No1: Los determinantes de la confianza en las instituciones

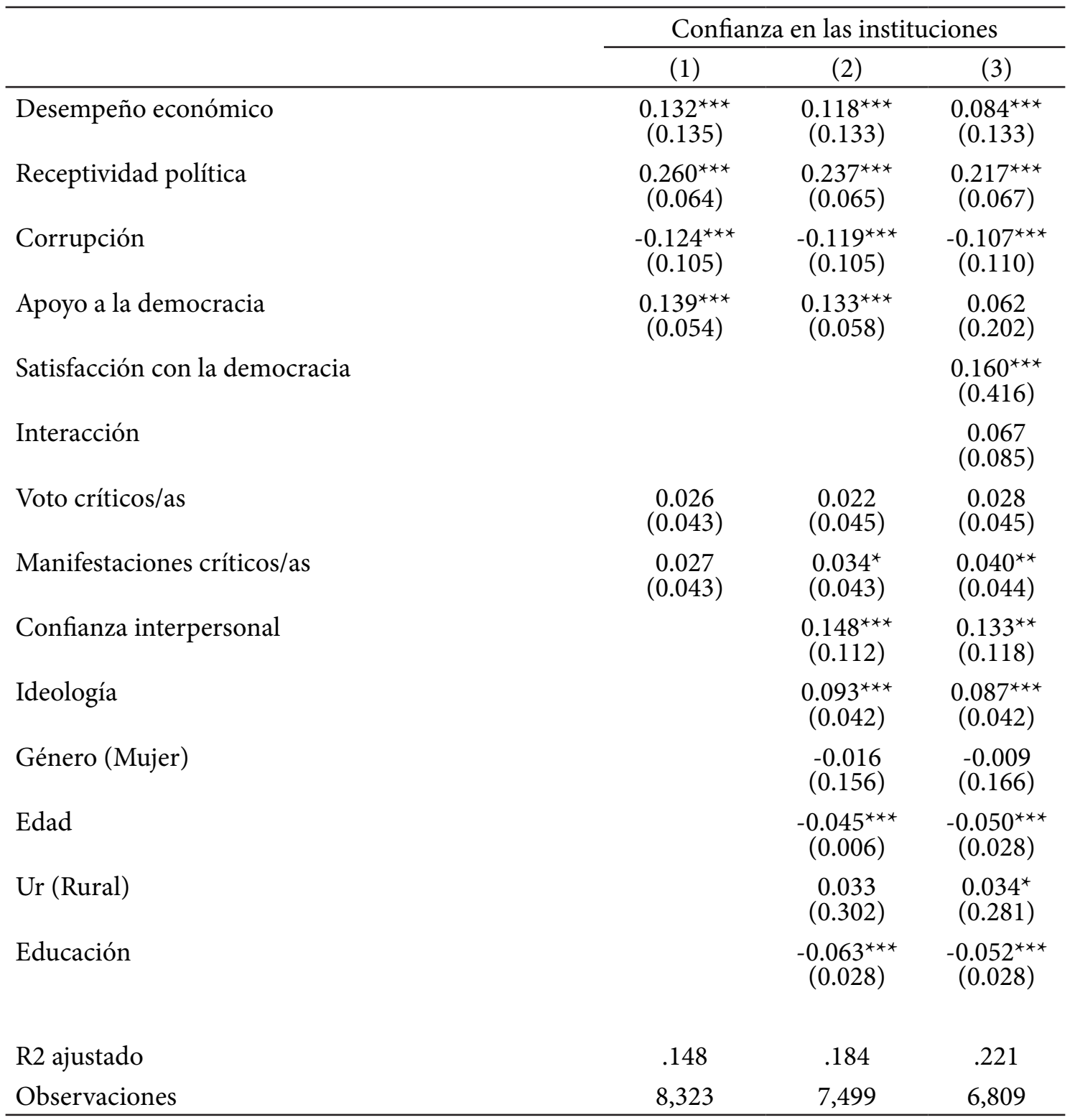

Errores estándar en paréntesis.

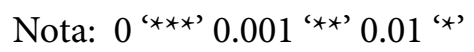

En un segundo nivel de análisis, los resultados de los modelos de regresión muestran que los impactos de las variables relacionadas a las características del proceso político también presentan impactos estadísticamente significativos, positivos y consistentes a lo largo de todos los modelos. De manera particular, el sentido de representación, o la percepción de la ciudadanía de que las autoridades políticas son receptivas de sus demandas y necesidades (Receptividad politica) es el predictor de mayor impacto sobre los niveles de confianza en las instituciones en términos comparados. El aumento de un punto (en desviaciones estándar) de esta variable, representa el incremento de .237 desviaciones estándar en la variable dependiente. 
Por su parte, y alineado con lo señalado por diferentes autores (Mishler y Rose, 2001; Catterberg y Moreno, 2006; Chang y Chu, 2006; Morris y Klesner, 2010), la percepción de que las y los funcionarios públicos se encuentran relacionados con la corrupción tiene impactos estadísticamente significativos y negativos en la confianza en las instituciones. El aumento de un punto en desviaciones estándar de la variable Corrupción disminuye la confianza en las instituciones en .119 desviaciones estándar. En consecuencia, estos resultados replican un sólido hallazgo de la discusión: la difundida percepción de corrupción conlleva consigo la idea de instituciones y autoridades despreocupadas por las necesidades de sus ciudadanos/as y desinteresadas de sus demandas, así como limitaciones en términos de resultados de política pública esperados. Por consiguiente, se vuelven objetos políticos poco fiables (van der Meer y Dekker, 2011; Morris y Klesner, 2010; Della Porta, 2000).

Pasando al plano de los valores democráticos, los resultados son menos claros que en el caso del desempeño institucional. En primer lugar, como lo muestran el Modelo 1 y el Modelo 2, el apoyo explícito a la democracia como forma de gobierno preferible (Apoyo a la democracia) tiene impactos estadísticos significativos, positivos y comparativamente importantes en el nivel de confianza institucional. El aumento de un punto (en desviaciones estándar) en este predictor eleva los niveles de confianza institucional en .133 desviaciones estándar. Sin embargo, la idea de que el apoyo a la democracia tiene impactos sobre esta forma de confianza en función a la percepción sobre el funcionamiento del régimen político (Mauk, 2020) no parece replicarse en el caso peruano de forma particular. Como lo muestra el Modelo 3, en el que se incluyen los efectos de interacción, dicha asociación no muestra significancia estadística.

A su vez, en relación con los predictores que evalúan el nivel de tolerancia política, no se muestran mejores resultados. El apoyo a la participación electoral de las personas con opiniones críticas a las del gobierno (Voto críticos/as) no muestra impactos estadísticamente significativos en la confianza en las instituciones. En el caso del apoyo a las manifestaciones pacíficas de estas personas (Manifestaciones críticos/as), esta variable sí presenta impactos estadísticamente significativos y positivos, pero el valor de su coeficiente es bajo en comparación a los demás predictores del modelo. Manteniendo todos los demás regresores constantes, el aumento de un punto en esta variable en desviaciones estándar eleva los niveles confianza en las instituciones en .034 desviaciones estándar.

En suma, si bien el apoyo explícito a la democracia sí eleva los niveles de confianza en las instituciones, en el caso peruano, no parece que este apoyo se encuentre mediado por la percepción sobre el funcionamiento del régimen político. Y, por el contrario, aunque se ha planteado teóricamente que el apoyo al ordenamiento político democrático tiene expresiones en los niveles de confianza en las instituciones (Easton, 1975) y se esperaría encontrar alguna asociación entre la presencia de valores democráticos y la confianza en las instituciones, los resultados no son concluyentes. En el caso de los indicadores de tolerancia política, el apoyo 
a la manifestación de personas críticas al gobierno tiene impactos positivos, pero comparativamente bajos; mientras que el apoyo a la participación electoral de estas mismas personas, mediante el voto, no muestra impacto alguno.

Un dato más a tomar en cuenta, si se observan los impactos de algunas variables de control, es que los niveles de confianza interpersonal tienen impactos no menores en los niveles de confianza institucional. En el modelo 2, el aumento de una desviación estándar de este predictor eleva los niveles de confianza en estos objetos políticos en .148 desviaciones estándar, impactos mayores al del desempeño económico de las instituciones o la percepción de corrupción en el sistema político. De esa manera, estos hallazgos difieren de conclusiones que plantean que la predisposición de las y los ciudadanos a confiar tiene impactos bajos o inexistentes sobre la confianza en las instituciones (Newton y Norris, 2018; Mishler y Rose, 2001).

\section{Explorando variaciones según el tipo de institución}

En términos teóricos, ha sido señalado que la confianza se deposita sobre alguien o algo en relación a funciones o dominios específicos sobre los cuales esta persona u objeto tiene capacidad de agencia (Levi, 1996; Levi y Stroker, 2000). Como tal, se defiende que la ciudadanía es capaz de identificar los ámbitos de competencia de las instituciones en particular; y que «mucho se aprende de explorar las dinámicas de confianza y apoyo hacia instituciones específicas, ya que, en la práctica, la evidencia sugiere que los ciudadanos/as distinguen entre una y otra» (Norris, 2011, p. 29). Sobre ese paraguas teórico, a continuación, se explora la manera en que las explicaciones exógenas y endógenas a la confianza institucional varían según el tipo de institución en juego.

Empezando por las variables del desempeño institucional y las características del proceso político, estos son los predictores con impactos estadísticamente sostenidos y positivos a lo largo de todos los modelos de regresión. No obstante, el grado de impacto sobre la confianza varía en cada institución. Como lo muestra la Tabla No 2, la percepción de un adecuado manejo de la economía nacional, por parte de las instituciones, tiene notables impactos en la confianza en el presidente de la república. El aumento de un punto en desviaciones estándar de este predictor eleva la confianza en .176 desviaciones estándar. El desempeño económico de las instituciones impacta positivamente en todas las instituciones a excepción de las Fuerzas Armadas, donde no muestra impactos significativos. Por su parte, sus impactos son moderados para el caso de las instituciones electorales (.061), los partidos políticos (.050) y los municipios distritales (.045); mientras que bastante más bajos para el caso del Congreso de la República (.028) y la policía (.025).

A nivel de proceso político, Receptividad política es el predictor de mayor impacto sobre la confianza en las diferentes instituciones en comparación con las demás 
variables del modelo. El hecho de que las personas perciban a sus autoridades políticas como receptivas e interesadas por sus necesidades y demandas, impacta casi el doble que el desempeño económico en, por ejemplo, la confianza en los partidos políticos (.151 desviaciones estándar). En general, los aumentos en la confianza son parejos y oscilan entre .124 y .163 desviaciones estándar, siendo ligeramente mayores para el caso de la policía, el congreso y el presidente de la república. La percepción de corrupción, de forma similar, presenta impactos estadísticamente significativos y negativos a lo largo de todos los modelos. Sus impactos son mayores para el caso de la confianza en el Congreso de la República (-.120), la policía (-.099) y los partidos políticos (-.086); pero menores para el caso de las Fuerzas Armadas y el presidente de la república.

Tabla No2: Los determinantes de la confianza institucional, según tipo de institución (sin efectos de interacción)

\begin{tabular}{|c|c|c|c|c|c|c|c|}
\hline & Congreso & Partidos & Presidente & $\begin{array}{l}\text { Municipa- } \\
\text { lidad }\end{array}$ & FFAA & Policía & Elecciones \\
\hline & (1) & (2) & (3) & $(4)$ & (5) & (6) & (7) \\
\hline $\begin{array}{l}\text { Desempeño } \\
\text { económico }\end{array}$ & $\begin{array}{l}0.028^{\star} \\
(0.028)\end{array}$ & $\begin{array}{c}0.050^{* * *} \\
(0.026)\end{array}$ & $\begin{array}{c}0.176^{\star * *} \\
(0.033)\end{array}$ & $\begin{array}{l}0.045^{* * *} \\
(0.029)\end{array}$ & $\begin{array}{c}0.014 \\
(0.033)\end{array}$ & $\begin{array}{l}0.025^{\star} \\
(0.031)\end{array}$ & $\begin{array}{c}0.061^{* * *} \\
(0.031)\end{array}$ \\
\hline $\begin{array}{l}\text { Receptividad } \\
\text { política }\end{array}$ & $\begin{array}{c}0.161^{\star * *} \\
(0.013)\end{array}$ & $\begin{array}{c}0.151^{\star * *} \\
(0.013)\end{array}$ & $\begin{array}{c}0.160^{* * *} \\
(0.015)\end{array}$ & $\begin{array}{c}0.139^{* * *} \\
(0.014)\end{array}$ & $\begin{array}{c}0.131^{* * *} \\
(0.014)\end{array}$ & $\begin{array}{c}0.163^{* * *} \\
(0.013)\end{array}$ & $\begin{array}{c}0.124^{* * *} \\
(0.015)\end{array}$ \\
\hline Corrupción & $\begin{array}{c}-0.120^{\star * *} \\
(0.025)\end{array}$ & $\begin{array}{c}-0.086^{\star * *} \\
(0.023)\end{array}$ & $\begin{array}{c}-0.055^{\star \star \star} \\
(0.024)\end{array}$ & $\begin{array}{c}-0.072^{* * *} \\
(0.025)\end{array}$ & $\begin{array}{c}-0.045^{\star *} \\
(0.027)\end{array}$ & $\begin{array}{c}-0.099^{* * *} \\
(0.027)\end{array}$ & $\begin{array}{c}-0.040^{* *} \\
(0.026)\end{array}$ \\
\hline $\begin{array}{l}\text { Apoyo a la } \\
\text { democracia }\end{array}$ & $\begin{array}{c}0.050^{* * *} \\
(0.013)\end{array}$ & $\begin{array}{c}0.062^{\star * *} \\
(0.013)\end{array}$ & $\begin{array}{c}0.086^{* * *} \\
(0.014)\end{array}$ & $\begin{array}{c}0.083^{* * *} \\
(0.015)\end{array}$ & $\begin{array}{c}0.056^{* * *} \\
(0.015)\end{array}$ & $\begin{array}{c}0.055^{\star * *} \\
(0.014)\end{array}$ & $\begin{array}{c}0.128^{\star * *} \\
(0.014)\end{array}$ \\
\hline Voto críticos/as & $\begin{array}{l}-0.009 \\
(0.010)\end{array}$ & $\begin{array}{c}0.019 \\
(0.010)\end{array}$ & $\begin{array}{c}0.019 \\
(0.010)\end{array}$ & $\begin{array}{l}0.039^{*} \\
(0.010)\end{array}$ & $\begin{array}{l}0.028 \\
(0.011)\end{array}$ & $\begin{array}{c}0.006 \\
(0.010)\end{array}$ & $\begin{array}{l}0.050^{\star *} \\
(0.011)\end{array}$ \\
\hline $\begin{array}{l}\text { Manifestaciones } \\
\text { críticos/as }\end{array}$ & $\begin{array}{c}0.000 \\
(0.009)\end{array}$ & $\begin{array}{c}0.012 \\
(0.009)\end{array}$ & $\begin{array}{l}0.028 \\
(0.010)\end{array}$ & $\begin{array}{l}0.018 \\
(0.010)\end{array}$ & $\begin{array}{l}0.046^{* *} \\
(0.011)\end{array}$ & $\begin{array}{l}0.039^{\star *} \\
(0.010)\end{array}$ & $\begin{array}{c}0.033 \\
(0.011)\end{array}$ \\
\hline $\begin{array}{l}\text { Satisfacción } \\
\text { democracia }\end{array}$ & $\begin{array}{c}0.137^{\star * *} \\
(0.035)\end{array}$ & $\begin{array}{c}0.137^{\star * *} \\
(0.030)\end{array}$ & $\begin{array}{c}0.171^{\star * *} \\
(0.032)\end{array}$ & $\begin{array}{c}0.134^{* * *} \\
(0.032)\end{array}$ & $\begin{array}{c}0.082^{* * *} \\
(0.036)\end{array}$ & $\begin{array}{c}0.125^{\star * *} \\
(0.033)\end{array}$ & $\begin{array}{c}0.161^{* * *} \\
(0.035)\end{array}$ \\
\hline $\begin{array}{l}\text { Confianza } \\
\text { interpersonal }\end{array}$ & $\begin{array}{c}0.057^{\star * *} \\
(0.026)\end{array}$ & $\begin{array}{c}0.048^{\star * \star} \\
(0.023)\end{array}$ & $\begin{array}{c}0.094^{\star * *} \\
(0.026)\end{array}$ & $\begin{array}{c}0.149^{* * *} \\
(0.029)\end{array}$ & $\begin{array}{c}0.073^{* * *} \\
(0.027)\end{array}$ & $\begin{array}{c}0.119^{* * *} \\
(0.026)\end{array}$ & $\begin{array}{c}0.095^{\star * *} \\
(0.025)\end{array}$ \\
\hline Ideología & $\begin{array}{c}0.074^{\star * *} \\
(0.008)\end{array}$ & $\begin{array}{c}0.061^{\star * \star} \\
(0.008)\end{array}$ & $\begin{array}{c}0.065^{\star * *} \\
(0.010)\end{array}$ & $\begin{array}{c}0.064^{* * *} \\
(0.010)\end{array}$ & $\begin{array}{l}0.029^{\star} \\
(0.010)\end{array}$ & $\begin{array}{c}0.059^{* * *} \\
(0.010)\end{array}$ & $\begin{array}{c}0.054^{\star * *} \\
(0.010)\end{array}$ \\
\hline Género (Mujer) & $\begin{array}{c}0.022 \\
(0.039)\end{array}$ & $\begin{array}{l}-0.013 \\
(0.036)\end{array}$ & $\begin{array}{l}-0.001 \\
(0.038)\end{array}$ & $\begin{array}{l}0.017 \\
(0.037)\end{array}$ & $\begin{array}{c}-0.063^{* * *} \\
(0.039)\end{array}$ & $\begin{array}{l}0.030^{*} \\
(0.041)\end{array}$ & $\begin{array}{c}-0.036^{* * *} \\
(0.035)\end{array}$ \\
\hline Edad & $\begin{array}{c}-0.068^{* * *} \\
(0.001)\end{array}$ & $\begin{array}{c}-0.075^{\star * *} \\
(0.001)\end{array}$ & $\begin{array}{c}0.025 \\
(0.001)\end{array}$ & $\begin{array}{l}-0.031^{*} \\
(0.001)\end{array}$ & $\begin{array}{c}-0.061^{* * *} \\
(0.002)\end{array}$ & $\begin{array}{l}-0.020 \\
(0.001)\end{array}$ & $\begin{array}{l}-0.004 \\
(0.001)\end{array}$ \\
\hline Ur (Rural) & $\begin{array}{c}0.057^{\star * *} \\
(0.055)\end{array}$ & $\begin{array}{c}0.033 \\
(0.056)\end{array}$ & $\begin{array}{c}0.009 \\
(0.064)\end{array}$ & $\begin{array}{c}0.002 \\
(0.081)\end{array}$ & $\begin{array}{c}0.017 \\
(0.065)\end{array}$ & $\begin{array}{l}-0.012 \\
(0.064)\end{array}$ & $\begin{array}{l}0.047^{\star *} \\
(0.063)\end{array}$ \\
\hline Educación & $\begin{array}{c}-0.069^{\star * *} \\
(0.006)\end{array}$ & $\begin{array}{c}-0.057^{\star * *} \\
(0.005)\end{array}$ & $\begin{array}{l}-0.009 \\
(0.006)\end{array}$ & $\begin{array}{c}-0.044^{\star *} \\
(0.007)\end{array}$ & $\begin{array}{c}-0.066^{* * *} \\
(0.006)\end{array}$ & $\begin{array}{c}-0.045^{\star *} \\
(0.006)\end{array}$ & $\begin{array}{l}0.030^{\star} \\
(0.007)\end{array}$ \\
\hline R2 ajustado & .115 & .099 & .161 & .114 & .072 & .105 & .122 \\
\hline Observaciones & 6,933 & 6,929 & 6,932 & 6,937 & 6,932 & 6,944 & 6,881 \\
\hline
\end{tabular}

Errores estándar en paréntesis.

Nota: $0^{(* * *)} 0.001^{(* *)} 0.01^{\text {(*) }}$ 
En conjunto, los hallazgos de los modelos muestran que, a nivel de desempeño institucional, la variable que tiene mayores impactos a la hora de explicar la confianza en las diferentes instituciones es la receptividad percibida desde la ciudadanía sobre sus autoridades políticas. Salvo por el caso del presidente de la república, donde el desempeño económico es el predictor con los coeficientes más altos, el grado de representación, como indicador característico del proceso político, muestra los mejores resultados. Así, aunque la confianza se otorgue sobre dominios específicos de acción, la representación política es un factor explicativo que «salpica» entre instituciones.

Pasando al análisis del impacto de los valores democráticos, lo primero que llama la atención es el impacto positivo y estadísticamente significativo y sostenido a lo largo de los modelos del apoyo explícito a la democracia como régimen de preferencia (Apoyo a la democracia). Comparativamente, estos son menores a los impactos de los predictores relacionados al proceso político, pero sí similares al del desempeño económico de las instituciones. El aumento de una desviación estándar en la variable independiente eleva la confianza en las instituciones electorales en .128 desviaciones estándar. En esa línea, los impactos se muestran mayores para el caso del presidente de la república (.086) y los municipios (.083).

Un hallazgo importante, además, es que la interacción propuesta entre el apoyo a la democracia y la percepción de su funcionamiento para explicar el impacto de los valores democráticos en la confianza institucional (Mauk, 2020) sí muestra evidencia, pero solo para el caso de la confianza en el presidente de la república. Como se observa en la Tabla No 3 (ver anexos), la interacción entre Apoyo a la democracia y Satisfacción democracia muestra impactos estadísticamente significativos en el Modelo 3. Así, los resultados indican que los efectos del apoyo a la democracia en la confianza en el presidente de la república son mayores para el caso de aquellos ciudadanos y ciudadanas que consideran que la democracia se encuentra operando de forma adecuada en el país (.147 desviaciones estándar). Para una interpretación más sencilla de los efectos de interacción, se encuentra en Gráfico No 2, donde se observa cómo el impacto del apoyo a la democracia en la confianza en el presidente depende del grado de satisfacción con el funcionamiento del régimen político. Los efectos de interacción, sin embargo, no muestran significancia estadística para el nivel de confianza en otra institución.

De vuelta con los demás predictores de tolerancia política, los resultados son sorpresivos. Primero, el apoyo a la participación electoral de las personas con opiniones críticas al gobierno (Voto críticos/as) presenta impactos estadísticamente significativos y positivos, solamente para el caso de la confianza en el municipio y las elecciones en el país. El aumento de un punto en desviaciones estándar de esta variable aumenta la confianza en ambas instituciones en .039 y 0.50 desviaciones estándar, respectivamente. 
Gráfico No 2: Efectos condicionales del apoyo a la democracia sobre la confianza en el presidente de la república, según el grado de satisfacción con el régimen político

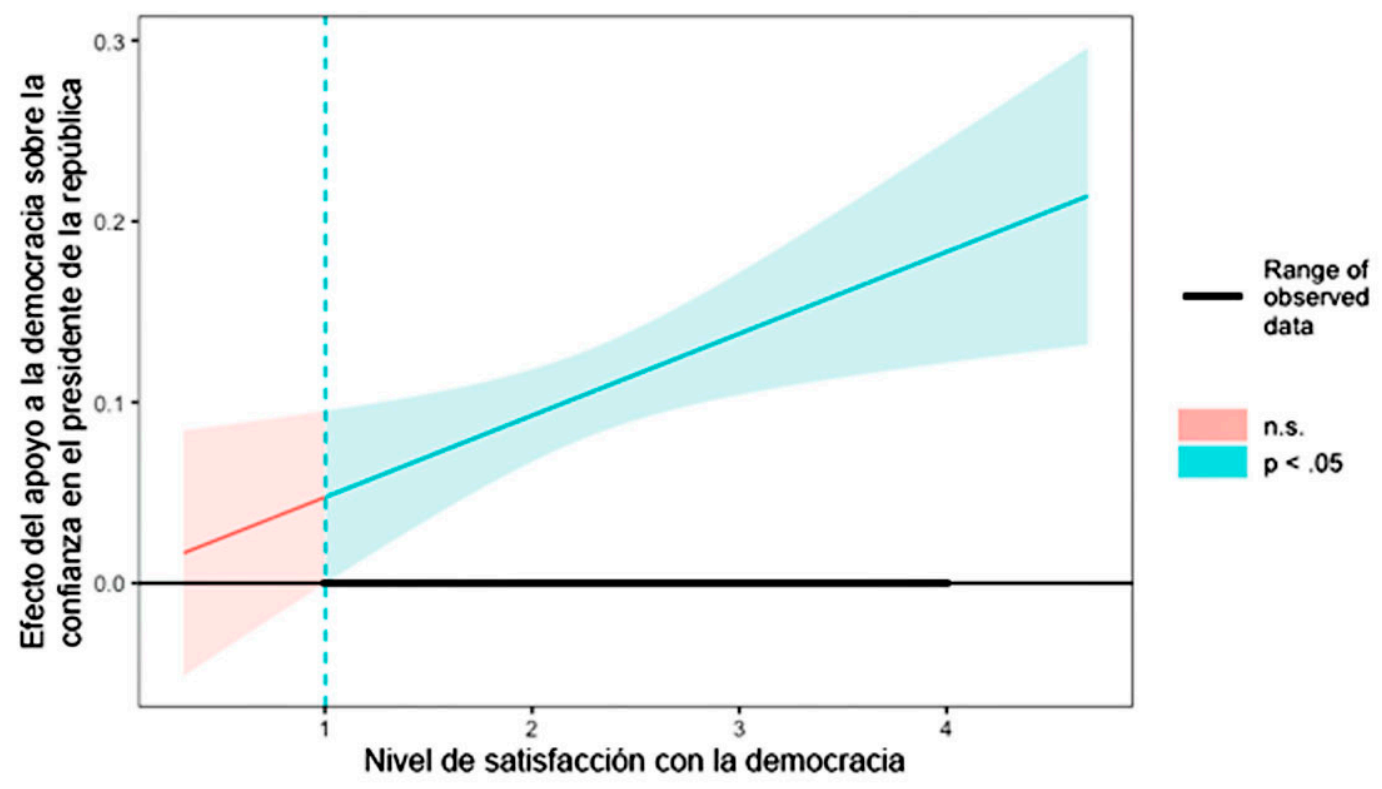

Elaboración propia. Fuente: Barómetro de las Américas (2010-2019)

Por el lado del apoyo a la manifestación pública y pacífica de estas personas (Manifestaciones críticos/as), curiosamente, la variable solo es estadísticamente significativa para el caso de las instituciones encargadas del uso de la fuerza y la seguridad interna. Si bien menores al caso de los demás predictores, el aumento de un punto (en desviaciones estándar) de esta variable eleva los niveles de confianza en las Fuerzas Armadas en .046, y la confianza en la policía en .039 desviaciones estándar. De esa manera, se observa que los impactos de los predictores relacionados a la tolerancia política se dan solamente en la confianza hacia algunas instituciones de forma específica.

Por último, es interesante analizar los impactos de algunas variables de control. Primero, el grado de satisfacción con la democracia tiene impactos importantes y sostenidos a lo largo de todos los modelos de regresión. Los impactos oscilan entre .082 y .137 desviaciones estándar frente al aumento de un punto en la variable independiente. De igual forma, el nivel de confianza interpersonal también presenta impactos sostenidos y positivos para todas las instituciones, aunque con impactos menores que en caso del grado de satisfacción con el régimen político.

Finalmente, y contrario a lo identificado por algunas investigaciones para el caso peruano (Arcaya, 2016), el nivel educativo presenta impactos inversos en los niveles de confianza en las instituciones a excepción de dos: la presidencia de la república y las elecciones. En el primer caso no presenta impactos estadísticamente significativos; mientras que, en el segundo, los impactos son positivos, aunque 
moderados: el aumento de un punto en desviaciones estándar eleva los niveles de confianza en las instituciones electorales en .030 desviaciones estándar.

\section{Conclusiones}

Varios trabajos en la literatura explican cómo la confianza en las instituciones es un elemento importante para el adecuado funcionamiento de la democracia. En la medida que este indicador expresa en alguna medida el grado de legitimidad que goza el proceso político (Almond y Verba, 1963) y la preferencia ciudadana por el ordenamiento democrático (Easton, 1975), los niveles altos de desconfianza en las instituciones políticas tienen repercusiones prácticas en el régimen: una menor disposición a cumplir las normas (Marien y Hooghe, 2011), bajos niveles de participación electoral (Hooghe, Marien y Pauwels, 2011) y el apoyo a propuestas políticas extremistas (Hooghe y Marien, 2013; van Stekelenburg y Klandersman, 2018).

Si bien los bajos niveles de confianza institucional es una problemática que existe en diferentes partes del mundo, el caso peruano se vuelve particularmente alarmante. Así, Perú no solo muestra niveles sumamente bajos de confianza en instituciones centrales como los partidos políticos o el Congreso de la República, sino que tampoco muestra mejores resultados en indicadores de tolerancia política, preocupación por el equilibrio de poderes o apoyo a la democracia (Carrión, Zárate, Boidi y Zechmeister, 2020). A pesar de ello, y aunque el país ha sido recurrentemente incluido dentro de conglomerados más grandes de casos para dar cuenta de los determinantes de esta forma de confianza, los esfuerzos por entender las variaciones en la confianza institucional, para el caso peruano de forma particular, han sido aún escasos.

Este trabajo buscó ocuparse precisamente de esa interrogante, recurriendo a los aportes teóricos ya desarrollados en la literatura para explorar los elementos que estarían detrás de las variaciones en la confianza en las instituciones, así como evaluar la manera en que estas macro-explicaciones presentan diferencias cuando se analiza el caso de cada tipo de institución en particular. En esa línea, se tomaron los valores democráticos y las evaluaciones sobre el desempeño institucional como explicaciones exógenas y endógenas al fenómeno de manera respectiva. Esta forma de aproximarse a las variaciones en la confianza en las instituciones, además, se alinea con un consenso relativamente extendido en los trabajos sobre cultura política: que en los individuos y grupos sociales coexisten valores políticos relativamente estables y difíciles de cambiar; junto con evaluaciones, actitudes y percepciones que son ilimitadas y mucho más fluctuantes (Aragón, 2010, p. 300).

Sin embargo, al momento de evaluar de qué forma interactúan ambos elementos para aproximarse a las variaciones en la confianza, se identifica que no parecen 
complementarse, sino más bien desarrollarse de manera independiente. Una de las principales conclusiones de este trabajo es que la confianza institucional se encuentra fuertemente asociada a las evaluaciones sobre el desempeño de estos objetos políticos, un hallazgo ya identificado por una variedad importante de estudios en la materia (Hetherington 1998; Mishler y Rose, 2001; Newton y Norris, 2018). Por el contrario, aunque el apoyo explícito a la democracia sí impacta positiva y sostenidamente en la confianza en las instituciones - pero en menor medida que el desempeño institucional—, los indicadores de tolerancia política no muestran los mismos impactos.

Adentrándose en estos resultados, además, se encuentra quela receptividad política, como evaluación sobre la calidad del proceso político en el sistema, muestra los impactos más altos y sostenidos, lo que lleva inevitablemente a discutir sobre la necesidad de representación y su importancia para elevar los niveles de confianza en las instituciones. Esto cobra una mayor relevancia si se toma en cuenta que, aunque no se puede hablar de una crisis generalizada de representación política en los regímenes democráticos, el caso peruano sostiene la clara continuidad de una situación deficitaria con relación a las percepciones de la ciudadanía sobre el grado de representación que encuentran en el gobierno, las autoridades y sus instituciones (Aragón, 2016, p. 134).

En consecuencia, mejorar el sentido de representación se vuelve un tema urgente. Pero, contradictoriamente, mejorarla puede llevar a la construcción de ciclos políticos poco virtuosos. Algunos trabajos muestran que mejorar estos sentidos de representación pasa por generar una sensación de mayor cercanía de las y los ciudadanos con el Estado y las autoridades, lo que abre la puerta a liderazgos políticos y gobiernos altamente populistas y de un compromiso dudoso con la democracia y sus instituciones (Mainwaring, Bejarano y Pizarro, 2006). Así, se estarían produciendo solo momentos de recuperación del «sentirse representado» y no una nueva forma de relacionamiento entre gobernantes y gobernados, que construya reservorios de legitimidad para las instituciones y soluciones a las problemáticas que aquejan a la ciudadanía en el largo plazo (Aragón, 2016).

Analizando algunas instituciones de forma particular, este trabajo muestra cómo, por ejemplo, la confianza en la figura del presidente de la república se encuentra relacionada de manera importante a la percepción de un adecuado manejo de la economía nacional. Asimismo, se pudo identificar cómo el apoyo a la manifestación pacífica de personas críticas impacta positivamente en la confianza de las instituciones encargadas justamente del uso de la fuerza, lo que no se replica para el caso de otras instituciones. De manera similar, el apoyo a la participación electoral de estas mismas personas solo tiene impactos en la confianza en las instituciones electorales y en los municipios. 
Por su parte, y contrario a lo señalado por varios trabajos (Mishler y Rose, 2001; Newton y Norris, 2018), la confianza entre pares es un predictor importante de la confianza en las instituciones. En consecuencia, se encuentra alguna evidencia en el caso peruano para el argumento teórico sobre la relación que existe entre las predisposiciones a confiar que se construyen en círculos sociales más pequeños y la confianza en las instituciones políticas. La institucionalización de patrones de interacción en estos círculos iniciales construye predisposiciones positivas que luego son proyectadas «hacia arriba», centrándose en estos objetos políticos (Putnam, 2000).

De otro lado, y contrario a lo planteado por algunos trabajos (Arcaya, 2016), los niveles educativos, más que elevar los niveles de confianza en las instituciones, en realidad presentan una relación inversa, mostrando una mayor sintonía con la literatura de los «ciudadanos críticos» (Norris, 2011). La excepción, sin embargo, ocurre cuando se trata de la confianza en las elecciones, donde se encuentran impactos significativos y positivos. Así, se aprecia que, como ya ha sido discutido por algunas investigaciones, la relación entre confianza institucional y el nivel educativo depende, fundamentalmente, de la manera en la que los individuos perciben y caracterizan a los objetos políticos de manera específica (Hakhverdian y Mayne, 2012).

A modo de cierre, los hallazgos de esta investigación muestran el sustancial impacto del desempeño percibido de las instituciones sobre esta forma de confianza, con énfasis en el sentido de representación. No obstante, estas asociaciones presentan variaciones importantes cuando se abordan los diferentes tipos de institución de forma específica. Así, por ejemplo, se ha podido observar cómo algunos valores políticos presentan impactos solamente cuando se trata de algunas instituciones de manera particular; o cómo algunas interacciones que son negativas en términos generales, como es el caso de los niveles educativos, muestran algunas excepciones para ciertos objetos políticos específicos. 


\section{Referencias bibliográficas}

Alessandro, M., Lagomarsino, B. C., Scartascini, C., Streb, J., \& Torrealday, J. (2021). Transparency and trust in government evidence from a survey experiment. World Development, 138, 105223. https://doi.org/10.1016/j.worlddev.2020.105223

Almond, G. A., \& Verba, S. (1963). The civic culture: Political attitudes in five western democracies. Princeton: Princeton University Press.

Aragón, J. (2010). Cultura política en perspectiva comparada. En Meléndez, C. \& Vergara, A. (Eds.), La iniciación de la política. El Perú político en perspectiva comparada (pp. 295324). Lima: Pontificia Universidad Católica del Perú.

Aragón, J. (2016). Representación política en los países andinos: crisis y recuperaciones. Revista de Ciencia Política y Gobierno, 3(5), 107-140.

Arcaya, L. E. (2016). Caracterización dela confianza en las instituciones del gobierno del Perú. (Tesis de Maestría. Universidad de Buenos Aires. Facultad de Ciencias Exactas y Naturales.). Recuperado de: http://hdl.handle.net/20.500.12110/tesis n6061 ArcayaArhuata

Blanco, L., \& Ruiz, I. (2013). The impact of crime and insecurity on trust in democracy and institutions. American Economic Review, 103(3), 284-88. DOI: 10.1257/aer.103.3.284

Breustedt, W., \& Stark, T. (2015). Thinking Outside the Democratic Box: Political Values, Performance and Political Support in Authoritarian Regimes: A Comparative Analysis. In Eder, C.; Mochmann, I. C. \& Quandt, M. (Eds.), Political Trust and Disenchantment with Politics (pp. 184-222). Leiden, The Netherlands: Brill.

Carrión, J. F., Zárate, P., Boidi, F., \& Zechmeister, E. J. (2020). Cultura democrática en Perú y las Américas, 2018/19: Tomándole el pulso a la democracia. Lima: USAID, Vanderbilt University, LAPOP \& IEP.

Catterberg, G., \& Moreno, A. (2006). The individual bases of political trust: Trends in new and established democracies. International Journal of Public Opinion Research, 18(1), 31-48. https://doi.org/10.1093/ijpor/edh081

Chang, E. C., \& Chu, Y. H. (2006). Corruption and trust: exceptionalism in Asian democracies? The Journal of Politics, 68(2), 259-271.

Citrin, J., \& Green, D. P. (1986). Presidential leadership and the resurgence of trust in government. British journal of political science, 16(4), 431-453. https://doi.org/10.1017/ $\underline{\text { S0007123400004518 }}$

Cruz, M., \& Guibert, Y. (2013). Determinantes de la confianza en el Congreso en el Perú. Cuaderno de Investigación No2. Instituto de Opinión Pública. Recuperado de: http:// repositorio.pucp.edu.pe/index/handle/123456789/33335

Dargent, E. (2021). El Páramo Reformista: Un Ensayo Pesimista sobre la Posibilidad de Reformar al Perú. Lima: Pontificia Universidad Católica del Perú.

Della Porta, D. (2000). Social capital, beliefs in government, and political corruption. In Pharr, S. J. \& Putnam, R. D. (Eds.), Disaffected democracies: What's troubling the trilateral countries (pp. 202-228). Princeton: Princeton University Press. 
Easton, D. (1965). A System Analysis of Political Life. Nueva York: Wiley.

Easton, D. (1975). A re-assessment of the concept of political support. British journal of political science, 5(4), 435-457. https://doi.org/10.1017/S0007123400008309

Fiorina, M. P. (1978). Economic Retrospective Voting in American National Elections: A Micro-Analysis. American Journal of Political Science, 22(2), 426-443. https://doi. org/10.2307/2110623

Freitag, M., \& Bühlmann, M. (2009). Crafting trust: The role of political institutions in a comparative perspective. Comparative Political Studies, 42(12), 1537-1566. https://doi. org/10.1177/0010414009332151

Fuchs, D. (2009). The Political Culture Paradigm. En Dalton, R. J. \& Klingemann H. D. (Eds.), The Oxford Handbook of Political Behavior (pp. 161-184). Oxford: Oxford University Press.

Gabriel, O. W. (1995). Political efficacy and trust. In van Deth, J. \& Scarbrough, E. (Eds.), The Impact of Values (pp. 357-390). Oxford: Oxford University Press.

Guëmes. C. (2015). Desconfianza en las administraciones públicas: ¿castigo por desempeño deficiente o prejuicio social? Análisis comparado en América Latina. XX Congreso Internacional del CLAD sobre la Reforma del Estado y de la Administración Pública. Lima, Perú. 10-13 nov. 2015.

Guëmes. C., \& Brugué, Q. (2016). When all I want is you. Confianza y Gobierno Abierto en América Latina. En Nasser, A., Ramírez-Alujas, A., \& Rojas, D. (Eds.), Desde el gobierno abierto al Estado abierto en América Latina y el Caribe (pp. 253-282). Santiago de Chile: Comisión Económica para América Latina y el Caribe (CEPAL).

Hardin, R. (1996). Trustworthiness. Ethics, 107(1), 26-42.

Hakhverdian, A., \& Mayne, Q. (2012). Institutional trust, education, and corruption: A micro-macro interactive approach. The Journal of Politics, 74(3), 739-750. https://doi. org/10.1017/S0022381612000412

Hetherington, M. J. (1998). The political relevance of political trust. American Political Science Review, 92(4), 791-808. https://doi.org/10.2307/2586304

Hetherington, M. J., \& Rudolph, T. J. (2008). Priming, performance, and the dynamics of political trust. The Journal of Politics, 70(2), 498-512. https://doi.org/10.1017/ $\underline{\text { S0022381608080468 }}$

Hooghe, M., \& Marien, S. (2013). A comparative analysis of the relation between political trust and forms of political participation in Europe. European Societies, 15(1), 131-152. https://doi.org/10.1080/14616696.2012.692807

Hooghe, M., Marien, S., \& Pauwels, T. (2011). Where do distrusting voters turn if there is no viable exit or voice option? The impact of political trust on electoral behavior in the Belgian regional elections of June 2009. Government and Opposition, 46(2), 245-273. https://doi.org/10.1111/j.1477-7053.2010.01338.x

Kołczyńska, M. (2020). Democratic values, education, and political trust. International Journal of Comparative Sociology, 61(1), 3-26. https://doi.org/10.1177/0020715220909881 
Levi, M. (1996). A state of trust. Firenze: European University Institute.

Levi, M., \& Stoker, L. (2000). Political trust and trustworthiness. Annual Review of Political Science, 3(1), 475-507.

Levitt, B. S. (2011). Institutional Trust and Congressional Autonomy in Latin America: Expectations, Performance, and Confidence in Peru's Legislature. Journal of Politics in Latin America, 3(2), 73-105. https://doi.org/10.1177/1866802X1100300203

Lipset, S. M. (1959). Some social requisites of democracy: Economic development and political legitimacy. American political science review, 53(1), 69-105. https://doi. org/10.2307/1951731

Mainwaring, S., Bejarano, A. M., \& Pizarro Leóngomez, E. (2006). The crisis of democratic representation in the Andes: An overview. En Mainwaring S.; Bejarano, A. M. \& Pizarro Leóngomez, E (eds.). The crisis of democratic representation in the Andes. Stanford: Stanford University Press.

Marien, S., \& Hooghe, M. (2011). Does political trust matter? An empirical investigation into the relation between political trust and support for law compliance. European Journal of Political Research, 50(2), 267-291. https://doi.org/10.1111/j.1475-6765.2010.01930.x

Mauk, M. (2020). Disentangling an elusive relationship: How democratic value orientations affect political trust in different regimes. Political Research Quarterly, 73(2), 366-380. https://doi.org/10.1177/1065912919829832

Medve-Bálint, G., \& Boda, Z. (2014). The poorer you are, the more you trust? The effect of inequality and income on institutional trust in East-Central Europe. Czech Sociological Review, 50(3), 419-454.

Miller, A., \& Listhaug, O. (1999). Political Performance and Institutional Trust. En: Norris, P (ed.) Critical Citizens: Global Support for Democratic Government. Oxford: Oxford University Press, pp.204-216

Mishler, W., \& Rose, R. (2001). What are the origins of political trust? Testing institutional and cultural theories in post-communist societies. Comparative Political Studies, 34(1), 30-62.

Morris, S. D., \& Klesner, J. L. (2010). Corruption and trust: Theoretical considerations and evidence from Mexico. Comparative political studies, 43(10), 1258-1285. https://doi. org/10.1177/0010414010369072

Norris, P. (2011). Democratic deficit: Critical citizens revisited. Cambridge: Cambridge University Press.

Newton, K., \& Norris, P. (2018). Confidence in Public Institutions: Faith, Culture, or Performance? In Pharr, S. J. \& Putnam, R. D. (Eds.), Disaffected democracies (pp. 52-73). Princeton: Princeton University Press.

OECD. (2013). Government at a Glance 2013. Paris: OECD Publishing. https://doi. org/10.1787/gov glance-2013-en. 
Paramio, L. (2015). Desafección política y gobernabilidad: el reto político. Madrid: Instituto de Estudios Latinoamericanos, Universidad de Alcalá, CAF-Banco de desarrollo de América Latina, Marcial Pons Ediciones Jurídicas y Sociales.

Putnam, R. D. (1995). Tuning in, tuning out: The strange disappearance of social capital in America. PS: Political science \& politics, 28(4), 664-683.

Putnam, R. D. (2000). Bowling alone: the collapse and revival of American community. New York: Simon and Schuster

Rahn, W. M., \& Rudolph, T. J. (2005). A tale of political trust in American cities. Public opinion quarterly, 69(4), 530-560. https://doi.org/10.1093/poq/nfi056

Rohrschneider, R., \& Schmitt-Beck, R. (2002). Trust in democratic institutions in Germany: Theory and evidence ten years after unification. German politics, 11(3), 35-58

Scartascini, C. \& Luna J. V. (2020). ¿En quién confiamos? Una cuestión de percepciones y desigualdad. En Busso, M., \& Messina, J. (Eds.), La crisis de la desigualdad. América Latina y el Caribe en la encrucijada (pp. 349-373). Banco Interamericano de Desarrollo.

Singh, S. P. (2018). Compulsory voting and dissatisfaction with democracy. British Journal of Political Science, 48(3), 843-854. https://doi.org/10.1017/S0007123416000041

Stevenson, B., \& Wolfers, J. (2011). Trust in public institutions over the business cycle. American Economic Review, 101(3), 281-87. DOI: 10.1257/aer.101.3.281

Taylor, M. A. (2000). Channeling frustrations: Institutions, economic fluctuations, and political behavior. European Journal of Political Research, 38(1), 95-134. https://doi. org/10.1111/1475-6765.00529

van der Meer, T. (2017). Democratic input, macroeconomic output and political trust. In S. Zmerli \& T. W. G. van der Meer (Eds.), Handbook of social trust (pp. 270-284). Cheltenham: Edward Elgar.

van der Meer, T., \& Dekker, P. (2011). Trustworthy states, trusting citizens? A multilevel study into objective and subjective determinants of political trust. In S. Zmerli \& M. Hooghe (Eds.), Political trust. Why context matters (pp. 95-116). Colchester, UK: ECPR Press.

van der Meer, T. W. G., \& Hakhverdian, A. (2017). Political Trust as the Evaluation of Process and Performance: A Cross-National Study of 42 European Countries. Political Studies, 65 (1), 81-102. https://doi.org/10.1177/0032321715607514

van Stekelenburg, J., \& Klandermans, B. (2018). In politics we trust... or not? Trusting and distrusting demonstrators compared. Political Psychology, 39(4), 775-792. https:// doi.org/10.1111/pops.12464 


\section{Anexo:}

Tabla No 3: Determinantes de la confianza institucional según tipo de institución (con efectos de interacción)

\begin{tabular}{|c|c|c|c|c|c|c|c|}
\hline & Congreso & Partidos & $\begin{array}{l}\text { Presiden- } \\
\text { te }\end{array}$ & $\begin{array}{l}\text { Munici- } \\
\text { palidad }\end{array}$ & FFAA & Policía & $\begin{array}{l}\text { Eleccio- } \\
\text { nes }\end{array}$ \\
\hline & $(1)$ & (2) & (3) & (4) & (5) & (6) & (7) \\
\hline $\begin{array}{l}\text { Desempeño } \\
\text { económico }\end{array}$ & $\begin{array}{l}0.029^{*} \\
(0.028)\end{array}$ & $\begin{array}{l}0.050^{\star * *} \\
(0.026)\end{array}$ & $\begin{array}{c}0.175^{\star * *} \\
(0.033)\end{array}$ & $\begin{array}{c}0.045^{\star * \star} \\
(0.029)\end{array}$ & $\begin{array}{l}0.014 \\
(0.033)\end{array}$ & $\begin{array}{c}0.025 \\
(0.031)\end{array}$ & $\begin{array}{c}0.061^{* * *} \\
(0.031)\end{array}$ \\
\hline $\begin{array}{l}\text { Receptividad } \\
\text { política }\end{array}$ & $\begin{array}{c}0.161^{* * *} \\
(0.013)\end{array}$ & $\begin{array}{c}0.151^{\star * *} \\
(0.013)\end{array}$ & $\begin{array}{c}0.161^{\star * *} \\
(0.015)\end{array}$ & $\begin{array}{c}0.139^{* * *} \\
(0.014)\end{array}$ & $\begin{array}{c}0.131^{* * *} \\
(0.014)\end{array}$ & $\begin{array}{c}0.163^{* * *} \\
(0.013)\end{array}$ & $\begin{array}{c}0.125^{\star * *} \\
(0.015)\end{array}$ \\
\hline Corrupción & $\begin{array}{c}-0.120^{* * *} \\
(0.025)\end{array}$ & $\begin{array}{c}-0.086^{* * *} \\
(0.023)\end{array}$ & $\begin{array}{c}-0.054^{\star * *} \\
(0.024)\end{array}$ & $\begin{array}{c}-0.072^{\star * *} \\
(0.025)\end{array}$ & $\begin{array}{c}-0.045^{\star *} \\
(0.027)\end{array}$ & $\begin{array}{c}-0.099^{\star * *} \\
(0.027)\end{array}$ & $\begin{array}{c}-0.039^{* *} \\
(0.026)\end{array}$ \\
\hline $\begin{array}{l}\text { Apoyo a la } \\
\text { democracia }\end{array}$ & $\begin{array}{c}0.078 \\
(0.041)\end{array}$ & $\begin{array}{c}0.011 \\
(0.038)\end{array}$ & $\begin{array}{l}-0.017 \\
(0.044)\end{array}$ & $\begin{array}{l}0.108^{*} \\
(0.047)\end{array}$ & $\begin{array}{l}0.011 \\
(0.054)\end{array}$ & $\begin{array}{c}0.016 \\
(0.052)\end{array}$ & $\begin{array}{c}0.069 \\
(0.050)\end{array}$ \\
\hline $\begin{array}{l}\text { Satisfacción con la } \\
\text { democracia }\end{array}$ & $\begin{array}{c}0.161^{* * *} \\
(0.090)\end{array}$ & $\begin{array}{l}0.092^{*} \\
(0.081)\end{array}$ & $\begin{array}{l}0.080^{*} \\
(0.094)\end{array}$ & $\begin{array}{c}0.156^{* * *} \\
(0.098)\end{array}$ & $\begin{array}{l}0.043 \\
(0.120)\end{array}$ & $\begin{array}{l}0.090^{*} \\
(0.105)\end{array}$ & $\begin{array}{l}0.109^{*} \\
(0.102)\end{array}$ \\
\hline Interacción & $\begin{array}{l}-0.039 \\
(0.018)\end{array}$ & $\begin{array}{l}0.072 \\
(0.017)\end{array}$ & $\begin{array}{l}0.147^{*} \\
(0.019)\end{array}$ & $\begin{array}{l}-0.036 \\
(0.020)\end{array}$ & $\begin{array}{l}0.065 \\
(0.022)\end{array}$ & $\begin{array}{l}0.057 \\
(0.022)\end{array}$ & $\begin{array}{c}0.085 \\
(0.021)\end{array}$ \\
\hline Voto críticos/as & $\begin{array}{l}-0.008 \\
(0.010)\end{array}$ & $\begin{array}{l}0.018 \\
(0.010)\end{array}$ & $\begin{array}{l}0.018 \\
(0.010)\end{array}$ & $\begin{array}{l}0.039^{*} \\
(0.010)\end{array}$ & $\begin{array}{l}0.027 \\
(0.011)\end{array}$ & $\begin{array}{c}0.006 \\
(0.010)\end{array}$ & $\begin{array}{l}0.050^{\star *} \\
(0.011)\end{array}$ \\
\hline $\begin{array}{l}\text { Manifestaciones } \\
\text { críticos/as }\end{array}$ & $\begin{array}{c}0.000 \\
(0.009)\end{array}$ & $\begin{array}{c}0.011 \\
(0.009)\end{array}$ & $\begin{array}{c}0.028 \\
(0.010)\end{array}$ & $\begin{array}{l}0.018 \\
(0.010)\end{array}$ & $\begin{array}{l}0.046^{* *} \\
(0.011)\end{array}$ & $\begin{array}{l}0.039^{* *} \\
(0.010)\end{array}$ & $\begin{array}{c}0.032 \\
(0.011)\end{array}$ \\
\hline $\begin{array}{l}\text { Confianza } \\
\text { interpersonal }\end{array}$ & $\begin{array}{c}0.057^{* * *} \\
(0.026)\end{array}$ & $\begin{array}{c}0.048^{\star * *} \\
(0.023)\end{array}$ & $\begin{array}{c}0.094^{* * *} \\
(0.026)\end{array}$ & $\begin{array}{c}0.149^{* * *} \\
(0.029)\end{array}$ & $\begin{array}{c}0.073^{* * *} \\
(0.027)\end{array}$ & $\begin{array}{c}0.118^{* * *} \\
(0.026)\end{array}$ & $\begin{array}{c}0.095^{\star * *} \\
(0.025)\end{array}$ \\
\hline Ideología & $\begin{array}{c}0.074^{* * *} \\
(0.008)\end{array}$ & $\begin{array}{c}0.062^{* * *} \\
(0.008)\end{array}$ & $\begin{array}{c}0.065^{\star * *} \\
(0.010)\end{array}$ & $\begin{array}{c}0.064^{* * *} \\
(0.010)\end{array}$ & $\begin{array}{l}0.029^{*} \\
(0.010)\end{array}$ & $\begin{array}{c}0.059^{* * *} \\
(0.010)\end{array}$ & $\begin{array}{c}0.055^{* * *} \\
(0.010)\end{array}$ \\
\hline Género (Mujer) & $\begin{array}{c}0.023^{* * *} \\
(0.039)\end{array}$ & $\begin{array}{l}-0.013 \\
(0.036)\end{array}$ & $\begin{array}{c}-0.002 \\
(0.038)\end{array}$ & $\begin{array}{c}0.018 \\
(0.037)\end{array}$ & $\begin{array}{c}-0.064^{* * *} \\
(0.039)\end{array}$ & $\begin{array}{l}0.029^{\star} \\
(0.041)\end{array}$ & $\begin{array}{c}-0.037^{* * *} \\
(0.034)\end{array}$ \\
\hline Edad & $\begin{array}{l}-0.068 \\
(0.001)\end{array}$ & $\begin{array}{c}-0.076^{* * *} \\
(0.001)\end{array}$ & $\begin{array}{c}0.023 \\
(0.001)\end{array}$ & $\begin{array}{l}-0.030^{*} \\
(0.001)\end{array}$ & $\begin{array}{c}-0.062^{* * *} \\
(0.002)\end{array}$ & $\begin{array}{l}-0.021 \\
(0.001)\end{array}$ & $\begin{array}{l}-0.004 \\
(0.001)\end{array}$ \\
\hline Urbano & $\begin{array}{c}0.057^{* * *} \\
(0.056)\end{array}$ & $\begin{array}{c}0.033 \\
(0.056)\end{array}$ & $\begin{array}{c}0.010 \\
(0.064)\end{array}$ & $\begin{array}{c}0.002 \\
(0.081)\end{array}$ & $\begin{array}{l}0.018 \\
(0.065)\end{array}$ & $\begin{array}{l}-0.011 \\
(0.064)\end{array}$ & $\begin{array}{l}0.048^{\star *} \\
(0.063)\end{array}$ \\
\hline Educación & $\begin{array}{c}-0.069^{* * *} \\
(0.006)\end{array}$ & $\begin{array}{c}-0.057^{* * *} \\
(0.005)\end{array}$ & $\begin{array}{l}-0.009 \\
(0.006)\end{array}$ & $\begin{array}{c}-0.044^{*} \\
(0.007)\end{array}$ & $\begin{array}{c}-0.066^{* * *} \\
(0.006)\end{array}$ & $\begin{array}{c}-0.045^{* *} \\
(0.006)\end{array}$ & $\begin{array}{l}0.030^{*} \\
(0.007)\end{array}$ \\
\hline R2 ajustado & .115 & .099 & .161 & .114 & .072 & .105 & .122 \\
\hline Observaciones & 6,933 & 6,929 & 6,932 & 6,937 & 6,932 & 6,944 & 6,881 \\
\hline
\end{tabular}

Errores estándar en paréntesis.

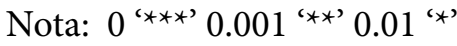

